\title{
Assessment of Regional Wall Motion Abnormality in Patients with Acute Anteroseptal ST Segment Elevation Myocardial Infarction
}

\author{
TUNAGGINA AFRIN KHAN ${ }^{1}$, SAIFUL AHMED ${ }^{2}$, MOSTASHIRUL HAQUE ${ }^{3}$, MD. RASUL AMIN ${ }^{3}$, \\ ATM IQBAL HASAN ${ }^{3}$, JAHANARA ARZU ${ }^{3}$, APARNA RAHMAN ${ }^{4}$, MD. ABU ZAHID \\ ${ }^{1}$ Department of Cardiology, United Hospital Limited, Dhaka, ${ }^{2}$ Department of Paediatric Surgery, Institute of Child and Mother \\ Health, Dhaka, ${ }^{3}$ Department of Cardiology, Bangabandhu Sheikh Mujib Medical University, Dhaka, ${ }^{4}$ Department of Cardiology, \\ BIRDEM General Hospital, Dhaka, ${ }^{5}$ Upazila Health Complex, Gangacara, Rangpur \\ Address of Correspondence: Dr. Tunaggina Afrin Khan, Department of Cardiology, United Hospital Limited, Dhaka, \\ E-mail: tunaggina.khan@gmail.com
}

\begin{abstract}
:
Post myocardial infarction (MI) short and long term clinical outcome is largely determined by the size of the infarcted area. It is generally assumed that as the lead involvement in the 12 lead electrocardiography (ECG) is less in anteroseptal ST segment elevation myocardial infarction (AS-STEMI), where ST segment elevation (STE) is limited to leads V1 to V3, myocardial damage is likely to be less. This study was intended to assess regional wall motion abnormality (RWMA) in acute anteroseptal STEMI patients. 90 patients with AS-STEMI admitted in between October 2012 and September 2013, were included. For each patient, a transthoracic echocardiogram (TTE) was performed within 24-48 hours of MI and was interpreted by an independent investigator blinded to the patient's ECG data.The mean ( \pm SD) age of the patients was 51.57 $( \pm 12.02)$ years with mean $( \pm S D)$ age of the patients was 52.58 ( \pm 12.02$)$ years with a range of $23-80$ years. There were $91.1 \%$ male and $8.9 \%$ female. The mean ( $\pm S D) E F \%$ was $38.80 \%( \pm 5.78)$. All the segments of left ventricle, except basal and mid inferolateral segments, were affected in anteroseptal STEMI. So, the term AS-STEMI may be a misnomer, as it implies that only the anteroseptal segments of the left ventricle are involved. This study shows that regional dysfunction in patients with AS-STEMI extends beyond the anteroseptal region and may be it is as much extensive as extensive anterior myocardial infarction. So, any patients with anterior wall involvement should be treated with utmost importance.
\end{abstract}

Key words: regional wall motion abnormality, anteroseptal STEMI.

University Heart Journal 2019; 15(1): 28-33

Introduction:

ST segment elevation myocardial infarction (STEMI) is the most lethal form of AMI. ${ }^{1}$ RWMA is one of the earliest features of acute MI even before the infarctive change is evident in standard ECG or by rise of cardiac biomarker. Echocardiography can detect RWMA and it is widely available, noninvasive and relatively cheap as well. ${ }^{1}$

Anterior ST segment elevation myocardial infarction (ASTEMI) involves the territory of the myocardium supplied by the major artery of the heart i.e. left anterior descending artery (LAD). It can be commonly classified as anteroseptal STEMI and extensive anterior STEMI. It is generally assumed that AS-STEMI is not a large MI and mainly anteroseptal region of left ventricle (LV) is affected. ${ }^{2-4}$
Conventionally, ECG leads are said to be oriented according to the anatomic zones of the left ventricle i.e. $\mathrm{V}_{1}-\mathrm{V}_{3}$ for anteroseptal zone, $\mathrm{V}_{4}-\mathrm{V}_{6}$ for apical or lateral zone, I and aVL for high lateral zone. However, these conventional electrodes cannot be pinpointed or placed directly upon the heart itself and are situated some distance away; therefore a large area of myocardial injury may be substantially attenuated on surface ECG. ${ }^{3}$

Shalevet al. ${ }^{5}$ suggested that, only the anterior-septum is never exclusively affected in patients with AS-STEMI. Porter et al. ${ }^{6}$ showed that STE in lead V1 and lead V2 is associated with different areas of infarction rather than septum. Bogaty et al. ${ }^{7}$ suggested that AS-STEMI is associated with an area of infarction predominantly involving the apex. But the apex may be involved either in anteroseptal, extensive anterior, or left dominant 
system occlusion related inferolateral MI. Bandeali et al. ${ }^{4}$ showed that AS-STEMI causes similar area of left ventricular involvement as that in EA-STEMI. Aldrich et al. ${ }^{8}$ suggested that the number of leads with ST segment elevation correlates with the infarct size in patients with A-STEMI, implying that AS-STEMI is associated with an infarct size smaller than that in patients with EA-STEMI. However neither angiographic nor echocardiographic data has correlated with either injury pattern with the expected location of injury. ${ }^{5-7,9}$

Two explanations regarding this matter opposing each other have been given; i.e. AS-STEMI is associated with a relatively small area of infarction, or it may be a manifestation of a large area of infarction, caused by a proximal occlusion of a wrapping left anterior descending artery (LAD) where STE in infero-lateral leads (leads V4 to V6) is cancelled by an opposing injury vector involving the basal segments, i.e. the antero-superior leads (leads V1 and V2). 5, 9-11

If MI was confined to the anterior septum, as it is thought, in so called AS-STEMI, it would require an isolated occlusion of the principal septal artery, and such an occurrence is unusual. An infarction of the anterior septum also would be expected to implicate occlusion of proximal LAD and should therefore be extensive. ${ }^{7}$ Zhong et al. ${ }^{10}$ found that STE in lead $V_{1}$ occurs when LAD is occluded proximal to first septal branch $\left(\mathrm{S}_{1}\right)$, which indicates that large area of myocardium is jeopardized which is perfused distal to $S_{1}$ and moreover, no significant difference was observed regarding the occlusion site between AS-STEMI and EA-STEMI in relation to the $S_{1}$ or first diagonal branch $\left(D_{1}\right)$ of LAD.

Whether AS-STEMI is truly an infarction involving smaller area of the left ventricular myocardium or it is more extensive, has not been well studied. The few studies carried out abroad were done with small sample size. This study was intended to assess echocardiographic RWMA in acute AS-STEMI patients with larger sample size.

\section{Materials and Methods:}

This cross sectional observational study was carried out in the Department of Cardiology, Dhaka Medical College Hospital, over a period of one year, from October, 2012 to September, 2013. By purposive sampling technique, 90 patients were selected. Anterior STEMI was defined as diagnostic new STE at the J point in at least 2 contiguous leads of $2 \mathrm{~mm}(0.2 \mathrm{mV})$ in men or $1.5 \mathrm{~mm}$ $(0.15 \mathrm{mV})$ in women in leads $\mathrm{V} 2-\mathrm{V} 3 \mathrm{and} /$ or of $1 \mathrm{~mm}$ $(0.1 \mathrm{mV})$ in other contiguous chest leads or the limb leads, provided there was no evidence of left ventricular hypertrophy or left bundle-branch block. ${ }^{12}$ AS-STEMI was denoted if STEMI was confined to leads $\mathrm{V}_{1}-\mathrm{V}_{3} \cdot{ }^{2,3,12}$
Patients with history of old myocardial infarction/ intracoronary intervention/ coronary artery bypass grafting, STE in ECG other than MI i.e. pericarditis, prinzmetal angina, Brugada syndrome etc., ECG evidence of LBBB, WPW syndrome, ventricular arrhythmia, advanced second degree or third degree conduction defect, ventricular electronic pacing of heart, patients with cardiomyopathy, valvular heart disease, severe co-morbid conditions such as ESRD, cirrhosis of liver, malignancy etc., patients who were not thrombolysed, and patients with poor echocardiographic windows were all excluded.

ECG was done immediately after admission and was interpreted by an expert cardiologist. TTE was done between 24 hours - 48 hours of MI by an expert echocardiographer blinded to the ECG diagnosis.

The left ventricle was divided into 17 segments ( 6 basal, 6 mid-ventricular, 5 apical). ${ }^{13}$ RWMA by TTE was assessed from each study subject according to the 17 segment model of left ventricle (Fig.1) in parasternal long axis, parasternal short axis, apical 2 chamber and apical 4 chamber views. RWMA detected in at least one segment was considered as involved and the results were recorded as normal, hypokinetic, akinetic and dyskinetic wall motion. Assessment of RWMA for each of the 17 segments was done. Global wall motion abnormality was also recorded as assessed on the basis of ejection fraction ( $\mathrm{EF} \%)$.

Data were expressed as mean $\pm \mathrm{SD}$ for continuous variables and as numbers (percent) for categorical variables. All statistical calculations were performed using SPSS for Windows (version 16).
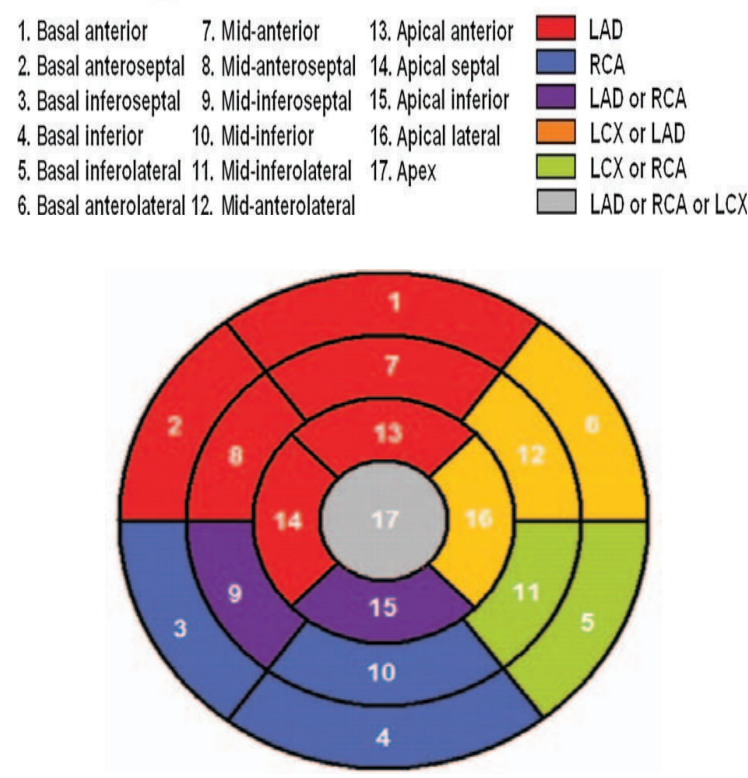

Fig.-1: 17 segment model of left ventricle with respective coronary circulation. ${ }^{13}$ 


\section{Results:}

The mean $( \pm \mathrm{SD})$ age of the patients was $52.58( \pm 12.02)$ years with a range of 23 - 80 years. $82(91.1 \%)$ patients were male and $8(8.9 \%)$ were female. Male and female ratio was almost 9:1 in the whole study subjects (Fig. 1).

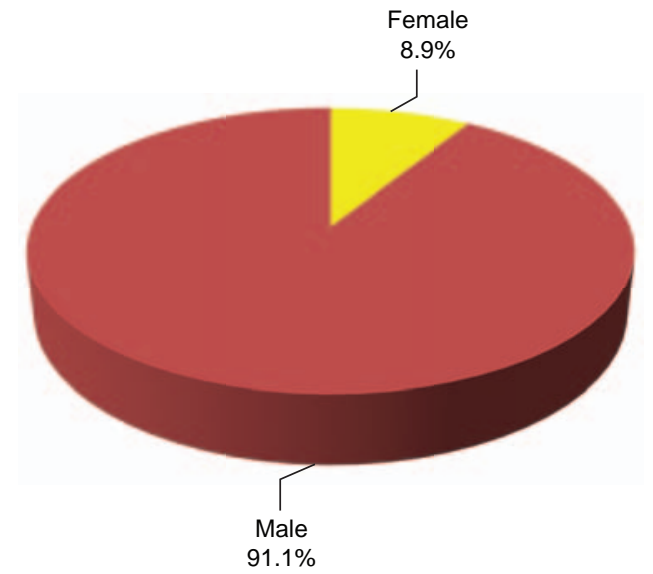

Fig.-2: pie chart showing gender distribution of the subjects.

Table-I

Traditional cardiac risk factors prevalence $(n=90)$

\begin{tabular}{lc}
\hline Traditional risk factors & $\begin{array}{c}\text { AS-STEMI }(\mathrm{n}=90) \\
\mathrm{n}(\%)\end{array}$ \\
\hline Smoking & $58(64.4 \%)$ \\
Hypertension & $42(46.7 \%)$ \\
Diabetes mellitus & $54(60.0 \%)$ \\
Dyslipidaemia & $76(84.4 \%)$ \\
Family history of CAD & $28(31.1 \%)$ \\
Sedentary life style & $68(75.6 \%)$ \\
Obesity & $20(22.2 \%)$ \\
\hline
\end{tabular}

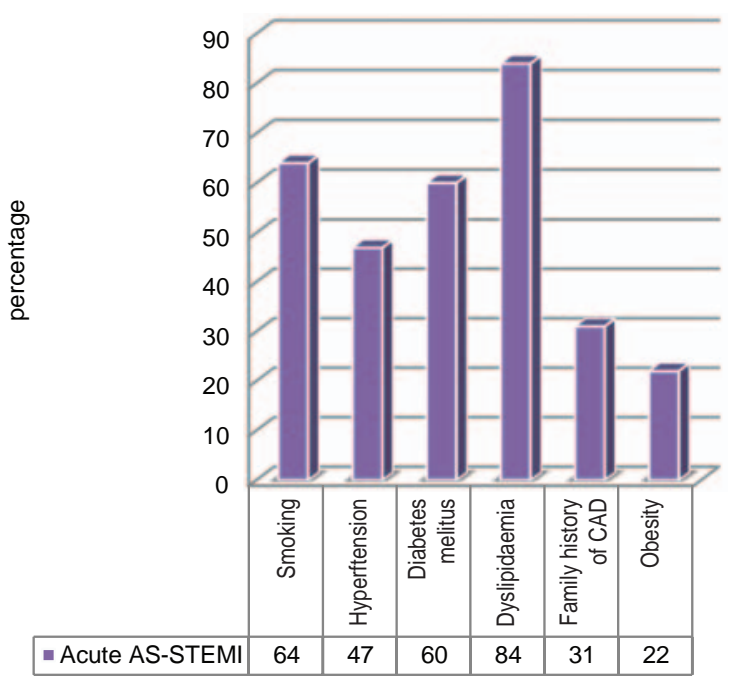

Fig.-3: Bar diagram showing distribution of traditional risk factors.
Table II

Assessment of biochemical paratmeters in AS-STEMI patients

\begin{tabular}{lc}
\hline & $(\mathrm{n}=90)$ \\
& mean \pm SD \\
\hline Troponin I (ng/ml) & $28.43 \pm 27.83$ \\
Fasting blood glucose level $(\mathrm{mmol} / \mathrm{L})$ & $7.36 \pm 2.88$ \\
Serum creatinine $(\mathrm{mg} / \mathrm{dl})$ & $1.46 \pm 0.49$ \\
Fasting lipid profile & \\
Total cholesterol $(\mathrm{mg} / \mathrm{dl})$ & $212.24 \pm 74.65$ \\
HDL cholesterol $(\mathrm{mg} / \mathrm{dl})$ & $38.49 \pm 11.37$ \\
LDL cholesterol $(\mathrm{mg} / \mathrm{dl})$ & $137.02 \pm 68.83$ \\
Triglyceride $(\mathrm{mg} / \mathrm{dl})$ & $191.02 \pm 69.89$ \\
\hline
\end{tabular}

More than $90 \%$ of the study subjects had predominant involvement of mid anterior, mid anteroseptal, mid inferoseptal, apical anterior, apical septal walls, and a significant number of study subjects had basal anterior, basal anteroseptal, apical anterior, apical septal segments involvement, indicating typical involvement of LAD territory. Variable degree of regional wall motion abnormality is seen in other segments indicating involvement of overlapping zones of LAD-RCA (right coronary artery) and LAD-LCX (left circumflex) artery supply (table III, IV).

\section{Table-III}

Wall motion pattern corresponding to the respective echocardiographic left ventricular segments

\begin{tabular}{llc}
\hline $\begin{array}{l}\text { Left ventricular segmental } \\
\text { wall motion }\end{array}$ & $(\mathrm{n}=90)$ \\
\hline Segment 1 & Normal & $\mathrm{n}(\%)$ \\
& Hypokinetic & $52(57.8 \%)$ \\
& Akinetic & $36(40.0 \%)$ \\
Segment 2 & Normal & $2(2.2 \%)$ \\
& Hypokinetic & $48(53.3 \%)$ \\
& Akinetic & $28(31.1 \%)$ \\
Segment 3 & Normal & $14(15.6 \%)$ \\
& Hypokinetic & $56(62.2 \%)$ \\
& Akinetic & $30(33.3 \%)$ \\
Segment 4 & Normal & $4(4.4 \%)$ \\
& Hypokinetic & $82(91.1 \%)$ \\
& Akinetic & $6(6.7 \%)$ \\
Segment 5 & Normal & $2(2.2 \%)$ \\
& Hypokinetic & $88(97.8 \%)$ \\
& Akinetic & $0(0.0 \%)$ \\
Segment 6 & Normal & $2(2.2 \%)$ \\
& Hypokinetic & $86(95.6 \%)$ \\
& Akinetic & $4(4.4 \%)$ \\
Segment 7 & Normal & $0(0 \%)$ \\
& Hypokinetic & $4(4.4 \%)$ \\
& Akinetic & $62(68.9 \%)$ \\
& & $24(26.7 \%)$ \\
\hline \multirow{5}{*}{ Sable continued }
\end{tabular}




\begin{tabular}{|c|c|c|}
\hline \multirow{3}{*}{ Segment 8} & Normal & $2(2.2 \%)$ \\
\hline & Hypokinetic & $38(42.2 \%)$ \\
\hline & Akinetic & $50(55.6 \%)$ \\
\hline \multirow[t]{3}{*}{ Segment 9} & Normal & $2(2.2 \%)$ \\
\hline & Hypokinetic & $40(44.4 \%)$ \\
\hline & Akinetic & $48(53.3 \%)$ \\
\hline \multirow{3}{*}{ Segment 10} & Normal & $74(82.2 \%)$ \\
\hline & Hypokinetic & $12(13.3 \%)$ \\
\hline & Akinetic & $4(4.4 \%)$ \\
\hline \multirow{3}{*}{ Segment 11} & Normal & $88(97.8 \%)$ \\
\hline & Hypokinetic & $2(2.2 \%)$ \\
\hline & Akinetic & $0(0 \%)$ \\
\hline \multirow{3}{*}{ Segment 12} & Normal & $34(37.8 \%)$ \\
\hline & Hypokinetic & $52(57.8 \%)$ \\
\hline & Akinetic & $4(4.4 \%)$ \\
\hline \multirow{3}{*}{ Segment 13} & Normal & $2(2.2 \%)$ \\
\hline & Hypokinetic & $58(64.4 \%)$ \\
\hline & Akinetic & $30(33.3 \%)$ \\
\hline \multirow[t]{3}{*}{ Segment 14} & Normal & $7(7.8 \%)$ \\
\hline & Hypokinetic & $34(37.8 \%)$ \\
\hline & Akinetic & $49(54.4 \%)$ \\
\hline \multirow[t]{3}{*}{ Segment 15} & Normal & $56(62.2 \%)$ \\
\hline & Hypokinetic & $26(28.9 \%)$ \\
\hline & Akinetic & $8(8.9 \%)$ \\
\hline \multirow[t]{3}{*}{ Segment 16} & Normal & $18(20.0 \%)$ \\
\hline & Hypokinetic & $62(68.9 \%)$ \\
\hline & Akinetic & $10(11.1 \%)$ \\
\hline \multirow[t]{3}{*}{ Segment 17} & Normal & $36(40.0 \%)$ \\
\hline & Hypokinetic & $38(42.2 \%)$ \\
\hline & Akinetic & $16(17.8 \%)$ \\
\hline
\end{tabular}

Table-III

Wall motion pattern corresponding to the respective echocardiographic left ventricular segments

\begin{tabular}{llc}
\hline $\begin{array}{l}\text { LV segmental wall motion } \\
\text { abnormality }\end{array}$ & $\begin{array}{c}(\mathrm{n}=90) \\
\mathrm{n}(\%)\end{array}$ \\
\hline Segment 1 & Basal anterior & $38(42.2 \%)$ \\
Segment 2 & Basal anteroseptal & $42(46.7 \%)$ \\
Segment 3 & Basal inferoseptal & $34(37.8 \%)$ \\
Segment 4 & Basal inferior & $8(8.9 \%)$ \\
Segment 5 & Basal inferolateral & $2(2.2 \%)$ \\
Segment 6 & Basal anterolateral & $4(4.4 \%)$ \\
Segment 7 & Mid anterior & $86(95.6 \%)$ \\
Segment 8 & Mid anteroseptal & $88(97.8 \%)$ \\
Segment 9 & Mid inferoseptal & $88(97.8 \%)$ \\
Segment 10 & Mid inferior & $16(17.8 \%)$ \\
Segment 11 & Mid inferolateral & $2(2.2 \%)$ \\
Segment 12 & Mid anterolateral & $56(62.2 \%)$ \\
Segment 13 & Apical anterior & $88(97.8 \%)$ \\
Segment 14 & Apical septal & $83(92.2 \%)$ \\
Segment 15 & Apical inferior & $34(37.8 \%)$ \\
Segment 16 & Apical lateral & $72(80.0 \%)$ \\
Segment 17 & Apical & $54(60.0 \%)$ \\
\hline
\end{tabular}

Table IV: presence of left ventricular segmental wall motion abnormality (hypokinetic, akinetic and dyskinetic wall motion) with respective echocardiographic left ventricular segment

In the present study, mean $( \pm \mathrm{SD})$ number of total involved segments was $8.83( \pm 2.49)$ with a range of $3-15$. The mean $( \pm \mathrm{SD})$ ejection fraction $(\mathrm{EF} \%)$ was $38.80 \%$ $( \pm 5.78)$.

\section{Discussion:}

Our finding was similar to Bandeali et al. ${ }^{4}$ who studied 65 subjects with anterior STEMI and mean age was 55.6 years with $59.6( \pm 12.7)$ years in AS-STEMI group. Porter et al. ${ }^{6}$ studied 58 patients with mean $( \pm \mathrm{SD})$ age of 61.5 $( \pm 14.6)$ years. Zafrir et al. ${ }^{14}$ studied 55 patients with mean $( \pm \mathrm{SD})$ age of $58.6( \pm 13.8)$ years. Huang et al. ${ }^{9}$ (2011) studied 97 patients with mean $( \pm \mathrm{SD}$ ) age of 63.4 $( \pm 12.0)$ years in AS-STEMI. Bandeali et al. ${ }^{4}$ had $68 \%$ male and $32 \%$ female in their study. Portal et al. ${ }^{6}$ had $76 \%$ male patients in their study and Zafrir et al. ${ }^{14}$ had $75 \%$ male in their study.

$64.4 \%$ of the our study population was smoker. Hypertension was present in $46.7 \%$ patients and type II diabetes mellitus was present in $60 \%$ patients. Dyslipidemia and positive family history for CAD were prevalent in $84.4 \%$ and $31.1 \%$ of the subjects. Sedentary life style and obesity were found in significant population ( $75.6 \%$ and $22.2 \%$ respectively).

In our study the mean $( \pm \mathrm{SD})$ ejection fraction $(\mathrm{EF} \%)$ was $38.80 \%$ ( \pm 5.78$)$. Bandeali et al. ${ }^{4}$ showed mean ( \pm SD) EF\% was $39.17 \%( \pm 10.5)$ in patients with AS-STEMI; moreover no significant difference in global wall motion abnormalities was found between patients with AS-STEMI and EA-STEMI assessed by mean EF. So, they concluded that the extent of damage in

AS-STEMI is more than usually thought. Portal et al. ${ }^{6}$ and Zafrir et al. ${ }^{14}$ also found similar results.

In the present study, mean $( \pm \mathrm{SD})$ number of total involved segments was $8.83 \pm 2.49$. This finding was partially supported by Bandeali et al. ${ }^{4}$ where mean number of total involved segments was $6.5 \pm 4$.

A trend was observed toward a greater degree of regional dysfunction in the basal-mid anterior, whole of the anteroseptal, inferoseptal, mid lateral and all apical regions. More than $90 \%$ of the study subjects had predominant involvement of mid anterior, mid anteroseptal, mid inferoseptal, apical anterior, apical septal walls, and a significant number of study subjects 
had basal anterior, basal anteroseptal segments, and apical anterior, apical septal segments involvement indicating typical involvement of LAD territory. Variable degree of regional wall motion abnormality seen in other segments indicates involvement of overlapping zones of LAD-RCA and LAD-LCX artery supply.

Our study was in agreement with Bandeali et al. ${ }^{4}$ They studied 65 patients with anterior STEMI who underwent TTE within 24-48 hours of admission. They also showed a significant proportion of the subjects having involvement of mid anterior, mid anteroseptal, mid inferoseptal, apical anterior, apical septal, true apical segments and a moderate number of study subjects having involvement of basal anterior, basal anteroseptal, mid to apical inferior segments. This finding was consistent with the current study. The apical segments involvement in AS-STEMI may be variable and this discrepancy can be explained by the presence of an alternative blood supply to the apical segments (because of the presence large diagonal or obtuse marginal branches or even branch of RCA), apical areas may be spared or involved in varying degree; moreover apex is an overlapping zone of LADRCA and LAD-LCX arteries. ${ }^{15}$

Huang et al. ${ }^{9}$ reported 2 contrasting angiographic findings and their relation to left ventricular segments of involvement among patients with A-STEMI. First, many patients with AS-STEMI than patients with EA-STEMI (52\% vs. $15 \%$ ) had proximal occlusion of a short LAD which did not reach the apex and an alternative blood supply to the apex by a large side branch, indicating more involvement of basal and mid segments sparing apical segments. In contrast, more patients with AS-STEMI than patients with EA-STEMI (52\% vs. 15\%) had a proximal occlusion of a long wrap-around LAD, suggesting extensive left ventricular area of involvement. This is consistent with the current study because of the similarities in wide range of involvement of left ventricular segments, variable involvement of apical segments. Their angiographic findings are supported by the echocardiographic findings of our study, suggesting AS-STEMI is not always limited to the anteroseptal segment.

Traditionally, it was believed V1 to V2 recorded the interventricular septum (hence the origin of the term anteroseptal). However, Shalev et $\mathrm{al}^{5}$ found that $92 \%$ of patients with STE in leads V1 to V3 had anteroapical infarction with no septal involvement. Similar findings were reported by Bogaty et al. ${ }^{7}$ Moreover, Porter et al. ${ }^{6}$ showed comparable global left ventricular function and regional dysfunction irrespective of the presence of STE in lead V5 or V6. No difference in regional dysfunction was observed between the patients with AS-STEMI and EA-STEMI showing similar involvement of basal anterior, basal anteroseptal, basal inferoseptal, and apical inferior segments between the anteroseptal and extensive anterior myocardial infarction patients without any statistical significance. Involvement of basal segments indicates proximal occlusion of LAD and extensive damage to left ventricle in both groups. All these evidences make it clear that each electrode records the global activation vector toward and away from each lead and not the local events adjacent to the electrode. Therefore, leads V1 to V3 do not represent only the anteroseptal regions. Therefore more sophisticated classification of anterior STEMI had been suggested. ${ }^{14}$

Zafrir et al. ${ }^{14}$ performed 17 segment based myocardial perfusion scan to see left ventricular segmental involvement in 55 patients with A-STEMI. The study showed involvement of basal segments occurring predominantly in AS-STEMI patients. This finding suggested more proximal occlusion of LAD in ASSTEMI.

Engelen et al. ${ }^{11}$ also studied 100 patients of acute anterior STEMI to find out the difference of occlusion site in AS-STEMI and EA-STEMI and found no difference in occlusion site between the groups. This finding also implied that AS-STEMI is not a MI involving areas less than EA-STEMI.

Conclusion: The present study concludes that RWMA and global LV dysfunction in ECG pattern of acute ASSTEMI is extensive. The term AS-STEMI neither implies that the ischemic process is limited to the anteroseptal segments, nor that the size of the ischemic area at risk is small. Therefore, the terminology "anteroseptal" is probably a misnomer and should not downgrade the extent of myocardial involvement

\section{References:}

1. Michael C. Kim, Annapoorna S. Kini, Valentine Fuster. Definitions of Acute Coronary Syndromes. In: Valentine Fuster, Richard A. Walsh, Robert A. Harrington, editors. Hurst's The Heart. $13^{\text {th }}$ ed. McGraw-Hill Medical Publisher, China; 2011. Chapter 56, p $1291,1293$.

2. Rationale and design of the GRACE (Global Registry of Acute Coronary Events) Project: a multinational registry of patients hospitalized with acute coronary syndromes. Am Heart J. 2001; 141(2): 190-9.

3. Leo Schamroth. An Introduction to Electrocardiography. $7^{\text {th }}$ ed. Blackwell science Publisher Ltd; 1990. P 15,17,132,136. 
4. Bandeali SJ, Stone S, Huang HD, Kayani WT, Wilson JM, Birnbaum Y. Comparison of segmental wall motion abnormalities on echocardiography in patients with anteroseptal versus extensive anterior wall ST-segment elevation myocardial infarction. J Electrocardiol. 2012; 45:551-55

5. Shalev Y, Fogelman R, Oettinger M, Caspi A. Does the electrocardiographic pattern of "anteroseptal" myocardial infarction correlate with the anatomic location of myocardial injury? Am J Cardiol. 1995;75:763-66.

6. Porter A, Wyshelesky A, Strasberg B, Vaturi M, Solodky A, Imbar $\mathrm{S}$ et al. Correlation between the admission electrocardiogram and regional wall motion abnormalities as detected by echocardiography in anterior acute myocardial infarction. Cardiology. 2000;94: $118-26$.

7. Bogaty P, Boyer L, Rousseau L, Arsenault M. Is anteroseptal myocardial infarction an appropriate term? Am J Med. 2002;113:3741.

8. Aldrich HR, Wagner NB, BoswickJ, Corsa AT, Jones MG, Grande P, et al. Use of initial ST-segment deviation for prediction of final electrocardiographic size of acute myocardial infarcts. Am J Cardiol. 1988;61:749-53.

9. Huang HD, Tran V, Jneid H, Wilson JM, Birnbaum Y. Comparison of angiographic findings in patients with acute anteroseptal versus anterior wall ST-elevation myocardial infarction. Am J Cardiol. 2011;107:827-32.

10. Zhong-qun Z, Wei W, Jun-feng W. Does left anterior descending coronary artery acute occlusion proximal to the first septal perforator counteract ST elevation in leads V5 and V6? J Electrocardiol. 2009; 42:52-57.

11. Engelen DJ, Gorgels AP, Cheriex EC, Muinck EDD, Ophuis AJO, Dassen WR, et al. Value of the electrocardiogramin localizing the occlusion site in the left anterior descending coronary artery in acute anterior myocardial infarction. J Am CollCardiol. 1999;34: 389-95.

12. ACCF/AHA Guideline for the Management of ST-Elevation Myocardial Infarction. 2013. Practice guideline. A Report of the American College of Cardiology. Foundation/American Heart Association Task Force on Practice Guidelines. Available from J Am Coll Cardiol.2013; 61(4): 78-140.

13. Cerqueira MD, Weissman NJ, Dilsizian V, Jacobs AK, Kaul S, Laskey WK, et al. Standardized myocardial segmentation and nomenclature for tomographic imaging of the heart: a statement for healthcare professionals from the Cardiac Imaging Committee of the Council on Clinical Cardiology of the American Heart Association. Circulation. 2002;105: 539-42.

14. Zafrir B, Zafrir N, Ben Gal T, Adler Y, Iakobishvili Z, Rahman MA et al. Correlation between ST elevation and Q waves on the predischarge electrocardiogram and the extent and location of MIBI perfusion defects in anterior myocardial infarction. ANE.2004; 9(2): $101-12$.

15. Birnbaum Y, Saeed M, Ware D. Correlation Between the Electrocardiogram and Regional Wall Motion Abnormalities as Detected by Echocardiography in Patient with Acute Anterior and Inferior Myocardial Infarction. Int J Bioelectromagn. 2003; 5(1): 1-3. 